\title{
Application of Digital Surgical Technique in Oral and Maxillofacial Trauma
}

\author{
Ping Chen, Xueli Zheng, Linglu Sun, Chenghua Ma, Zhiqiang Wang, \\ Yali Liu, Lijuan Ma, Ruoyu Wang, Lan Yang \\ School of stomatology Lanzhou Univeristy, Lanzhou 730000, China; \\ Email: chenping5685@163.com
}

Keywords: Digital surgery, Oral and maxillofacial, Trauma, Application.

\begin{abstract}
As a product of modernization, digital surgical technique integrates modern imaging technique, stereospecific technique, electronic computer technique, artificial intelligence technique and medicine. Hence, this technique has received much attention at home and abroad since it was created. In this paper, several related techniques are mainly utilized to analyze its application and development in oral and maxillofacial surgery and put forward the corresponding measures.
\end{abstract}

\section{Introduction}

At present, oral and maxillofacial digital surgery focuses mainly on two aspects, i.e. combination of individual digital design and rapid prototyping technique and application of surgical navigation. The two aspects show different features, which are complementary to each other, but cannot replace each other. This paper further explores the application value and development focus of digital surgery in oral and maxillofacial surgery based on the past theoretical and clinical studies.

\section{Individual Digital Design and Rapid Prototyping Techniques}

The two techniques were first reported at the beginning of the 1990s. For the rapid development of computer technology and medical imaging technique ever since, individual digital design and rapid prototyping techniques have been gradually applied in oral and maxillofacial surgery, neurosurgery, plastic surgery and other branches of medicine.

\subsection{Application in Preoperative Diagnosis and Surgical Design}

In terms of structure, oral and maxillofacial anatomy is originally complex, but individual digital design and rapid prototyping techniques can be used to process the three-dimensional skull simulation model with high accuracy. Currently, rapid prototyping technique is mainly divided into stereolithography prototyping, fused deposition prototyping, computerized mill, selected laser sintering, and laminated object manufacturing. Different technical processes are applicable to processing of different materials. By means of corresponding physical model, clinical doctors can have very intuitive knowledge about the actual condition of the operating area. For some relatively complicated cases, surgical simulation can be carried out with the assistance of the model to anticipate all kinds of problems in operation and identify the advantages and disadvantages of different operations. Also, the model can be employed in operation teaching and practice.

\subsection{Application in Individual Repair of Maxillofacial Defect}

For trauma, tumor or other factors, maxillofacial defect has tremendous impacts and effects on the facial form of patient, directly affecting the patient's quality of life, and it is difficult to treat to some extent. Traditional treatment relies mainly on free bone transplant, vascularized bone transplant, and artificial bone substitute implant, etc., which often faces some problems in practice. For instance, it is difficult to shape the bent bone flap, while the recovery of facial form is not satisfactory. After operation, the masticatory recovery of patient is unsatisfactory. Moreover, the duration of operation is extended. Rapid prototyping technique is utilized to prepare the high-precision three-dimensional 
model based on the shape of a patient's missing maxillofacial tissues, and the design of implant with casting technique is used with the model to realize the individual repair of defect.

In the study conducted by Silva et al., a three-dimensional skull model was fabricated with stereolithography prototyping, and used to simulate the process of operation and repair the complicated maxillofacial defect for 28 patients, which were all satisfied with the results. In his study, Liu Yanpu made the resin model with defective lower jaw by employing the CT scanning data and rapid prototyping technique, and employed the casting technique to fabricate the titanium repair framework, which were used in the individual repair for 4 patients having massive lower jaw defect after amputation of massive lower jaw lesion. After operation, facial form and masticatory function recovered satisfactorily as shown in the following figures.

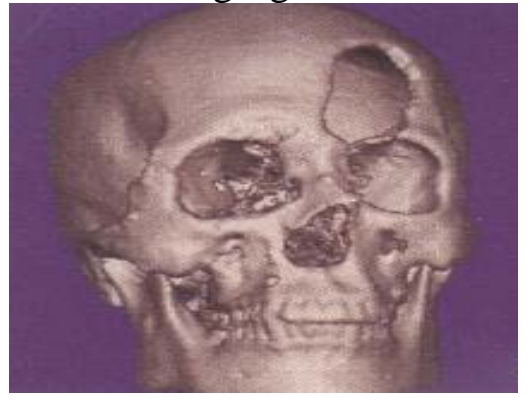

Figure 1 Three-dimensional reconstruction

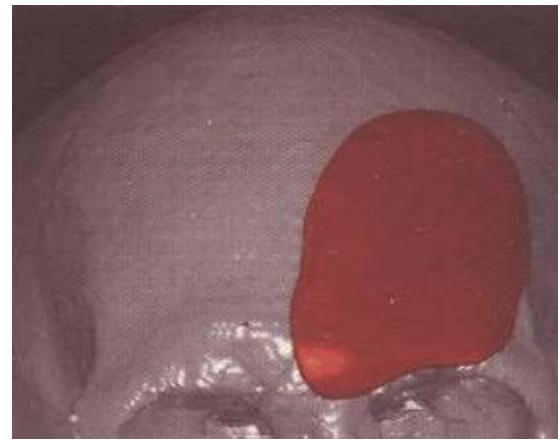

Figure 2 Design of individual implant

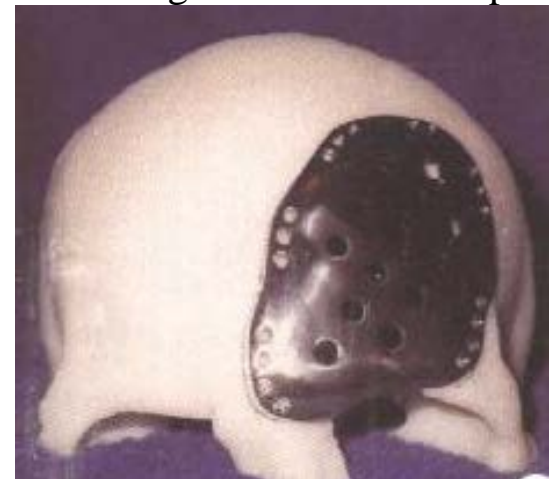

Figure 3 Three-dimensional model prefrabicated implant and simulation of pre-operation

\subsection{Application in Orthognathic Surgery}

In an operation of orthognathic surgery, accurate pre-operative design plays a very important role, but it is always very difficult to correct the asymmetric facial deformity for different reasons. In the past studies, Robiony utilized the three-dimensional CT data and rapid prototyping technique with accurate pre-operative design to perform an operation for one 9-year-old patient, which achieved the satisfying recovery of facial form and occluding relation subsequently. In the clinical experiment, CT visualization technology was employed to treat patients. First, the technology was utilized to construct a three-dimensional skull model and simulate the range of osteotomy and the motion of amputated bone section with the computer software. Subsequently, rapid prototyping technique could help make the physical model for the jaw of patient. The simulated operation on the model could achieve the remarkable results as well. 


\subsection{Application in Repair of Maxillofacial Trauma}

In this study, CT scanning data and three-dimensional CT surface are combined to present and analyze the relationship between orbital volume and enophthalmos of a patient suffering from orbital blowout fracture with 3DMSR. On this basis, individual digital design is employed to repair the peri-orbital defect, which is an objective proof that individual digital design and rapid prototyping technique can effectively prevent the future enophthalmos in a very remarkable manner.

\section{Surgical Navigation System}

In recent years, a new page is turned in the medical field thanks to the rapid development of medical imaging technique and the wide use of image processing technology and robot technology. In this field, surgical navigation system plays a very important role. In the middle and late 19th century, it was gradually applied in the neurosurgical operation, and then spread into other fields. The surgical navigation system has widened the visual range of surgeons to a large degree and burst the bounds of traditional surgical operation.

\subsection{Development of Surgical Navigation System}

Surgical navigation system can be regarded as an important branch of computer-aided surgery. In this system, all kinds of medical image information is utilized to navigate the important operating instruments and know about the position to be operated in an all-round way, so as to realize the pre-operative and intra-operative interactions regarding medical image, dissected position and operating area.

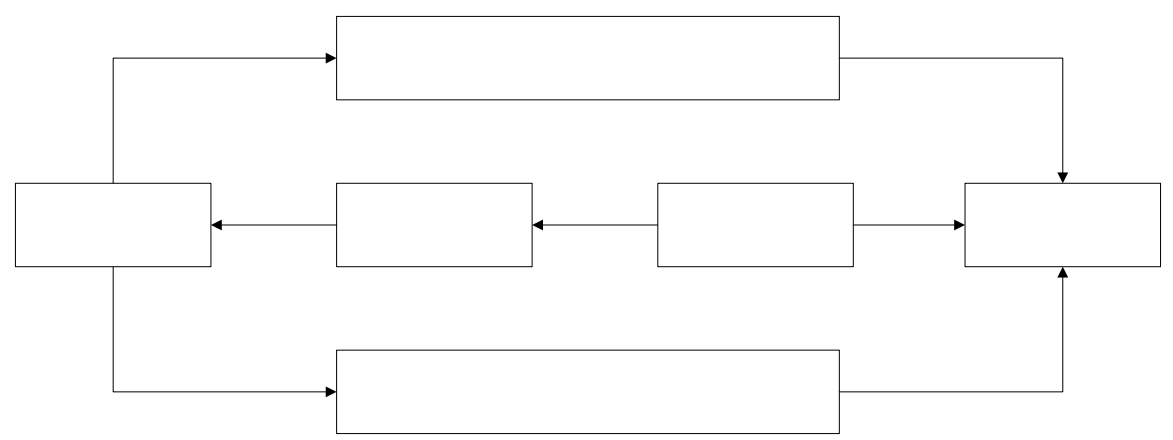

Figure 4 Navigation system structure

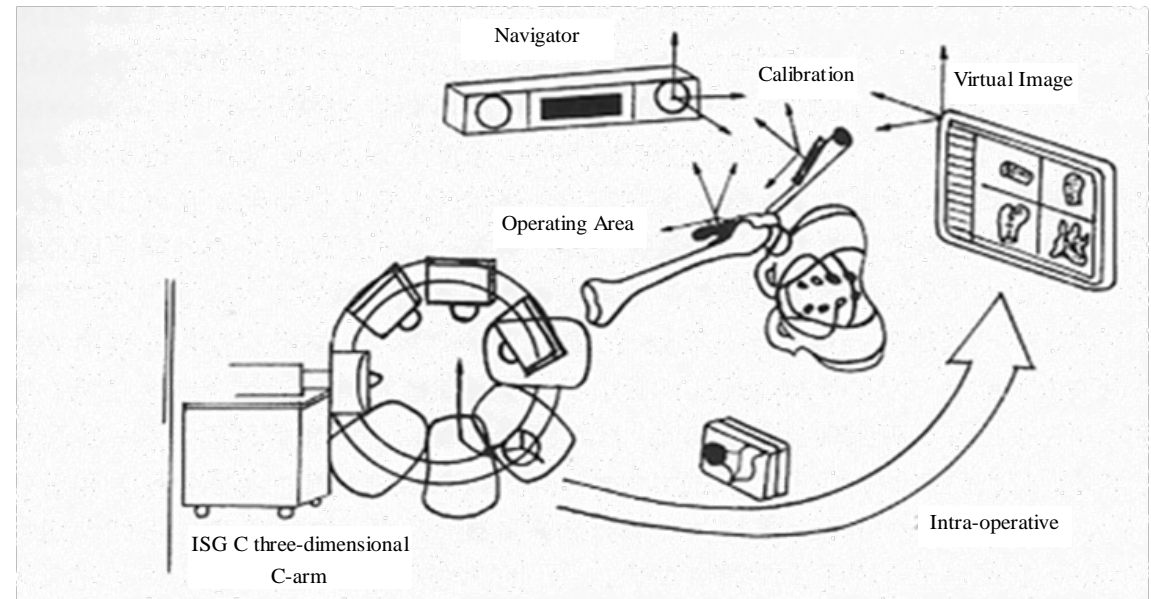

Figure 5 Optical navigation and positioning

In the medical field, surgical navigation system was first practiced in neurosurgery, and then further expanded and applied in other sectors. Meanwhile, computer-aided navigation system was correspondingly developed. The early studies mainly focused on the CT navigation for operation. However, fluoroscopic navigation system has been developed together with the open surgical navigation system without any anatomical image today. As revealed in their studies, foreign scholars focus mainly on the head X-ray examination and tooth model, and take it as the basis to construct the new navigation system. With regard to accuracy, it can satisfy the needs of operations for oral implantation, tumor removal and trauma repair. Also, some scholars employ the photoelectrical 
three-dimensional imaging technique to directly display the information of the operating area in their studies, which does not need the traditional way of implanting the mark into the head of patient. Hence, it can avoid the error caused by mark, so it enjoys a bright prospect for development.

\subsection{Application of Surgical Navigation System in Maxillofacial Tumor}

To tumor patient, the scope of surgical removal is very important. Based on the past practical results, surgical navigation system can visualize the local anatomy of lesion, so as to improve the quality of operation to a large degree. It is worth mentioning that the real-time navigation in operation enables the surgeon to excise the lesion strictly within the excision scope determined before operation, so as to avoid the important anatomy more accurately and effectively and improve both efficiency and safety of operation to some level. In general, surgical navigation system is applied to achieve very good results of operation when it is conducted strictly based on the scope of excision determined before operation.

\subsection{Application of Surgical Navigation System in Maxillofacial Trauma}

In traditional operations, the reduction of maxillofacial fracture depends much on the clinical experience of the surgeon, which restricts the accuracy of alignment and the functional recovery after operation. For instance, orbital fracture cannot be accurately reduced due to its subtle and complicated anatomy if the reduction relies only on the experience of the surgeon. After the navigation system is applied in the orbital region, it can remarkably improve the situation. In their studies, foreign scholars have pointed out that the application of surgical navigation system in maxillofacial trauma can have different materials for different degrees or positions of fracture, and shape the materials. Some doctors employ the point-to-point method to further realize the interaction between virtual operating model and actual operation, and treat the fracture at cheekbone and zygomatic arch. The method is very simple, but its results are very noticeable.

\subsection{Application of Surgical Navigation System in Maxillofacial Deformity}

For patients with congenital deformity, it is necessary to first obtain the original data in the study and analysis. The K-means cluster algorithm is utilized to segment the image and then establish a visualization model, which creates a basis for virtual operation. The virtual object tactile instrument can simulate the orthognathic surgery, and is combined with computer technology to realize the three-dimensional orthognathic surgical simulation platform, in order to achieve the random excision on the three-dimensional jaw model in the manner of human-computer interaction, and correct the deformity by means of fixed motion or rotating jaw.

In the repair process of maxillofacial deformity, a reference position is very important. In the study, Kau utilized a laser scanner to collect the facial impacts of 70 children, so as to generalize the facial features of children at the specific age. In this way, the facial features can be used to identify the facial deformity or abnormality of children at that age, which is difficult to notice under naked eyes.

\subsection{Application of Surgical Navigation System in Oral and Maxillofacial Distraction Osteogenesis}

In recent years, surgical navigation system has been further studied and practiced in the secondary distraction osteogenesis for jaw reconstruction and the vertical traction osteogenesis for alveolar atrophy after resection of tumor, etc. Also, it is still expanding into other fields. The distraction vector is affected by multiple factors, including direction of tractor axis, installation position of tractor, and tension of soft tissues in surrounding muscle. Before operation, it is therefore often difficult to predict the direction of distraction, which affects the final results of treatment as well. For distraction osteogenesis, surgical navigation system can first scan the front and lateral X-ray films of skull positioning, and upload them into the system to determine at least seven reference marking points, which are surgically positioned exactly as the positioning of X-ray films. In this way, fixed section and tractive section are connected to two sensing locators respectively. With electromagnetic and optical tracking system, the three-dimensional motion of tractive bone section on X-ray film can be also monitored on the computer screen to adjust and select the optimal traction vector during and after operation, so as to guarantee the effect of traction after operation. 


\section{Prospects}

Now, individual digital technique and rapid prototyping technique have been gradually applied in the oral and maxillofacial surgery, and will enjoy a broad space for development. Regardless of its problems and errors in application, rapid prototyping technique is expanding and developing continuously and plays an important role in the development of oral and maxillofacial surgery. In addition to the further development of surgical navigation system, its application in oral and maxillofacial surgery will be further expanded. Thanks to its noticeable features, surgical navigation system has burst the bounds of traditional surgical operation and redefine the concepts of surgical operation and surgical operating instruments, which is of great significance to improving the accuracy of operation positioning, shortening the duration of operation and enhancing the success rate of operation, etc. In future, digital technology will become an indispensable clinical auxiliary instrument for oral and maxillofacial surgery. Especially, the digital platforms including digital image transmission and storage, assistive diagnosis software and surgical design will help digital technology facilitate the progress of traditional medicine. In the near future, telemedicine, computer/robotics-aided surgery and navigation surgery will develop with digital medicine to gradually become new choices for surgical treatment.

\section{References}

[1]. Tian Weidong. Application of digital surgical technique in repair and reconstruction of oral and maxillofacial tissues. The First Global Congress of Chinese Dentists \& China International Stomatology Conference 2010. 2013.

[2]. Liu Xiaojing, He Yang, Gong Xi, et al. Application of computer navigation technology in oral and maxillofacial trauma repair. Chinese Journal of Stomatology, 2012, 47(11):645-650.

[3]. Li Zubing. Application of digital surgical technique in oral and maxillofacial trauma. Chinese Journal of Stomatology, 2013, 47(11):641-644.

[4]. Tian Weidong. Application of digital surgical technique in repairing secondary deformity of craniomaxillofacial trauma. Chinese Journal of Stomatology, 2014, 47(8):470-473.

[5]. Tang Wei. Application and prospect of oral and maxillofacial digital surgery. Journal of Oral and Maxillofacial Surgery, 2015, 18(6):381-385.

[6]. Xu Hanmei. Study on the application of plastic and aesthetic surgical technical principles in treatment of oral and maxillofacial trauma. China Medical Cosmetology, 2015(5):33-34.

[7]. Zheng Hai. Progress in application of absorptive materials in oral and maxillofacial trauma. Chongqing Medicine, 2013, 40(18):1845-1847.

[8]. Huo Junfeng, Yang Bei. Clinical experience of plastic and aesthetic surgical technical principles in treatment of oral and maxillofacial trauma. Medical Aesthetics and Beauty, 2015(3):188-188.

[9]. Jiang Lijian, Li Weiguo, Wu Xinzhong et al. Application of aesthetic principles in treatment of oral and maxillofacial trauma. China Medical Cosmetology, 2014, 13(4):436-437.

[10]. Zhang Zhiguang. Application of minimally invasive technique in oral and maxillofacial trauma. China Symposium on Repair and Reconstruction of Oral and Maxillofacial Trauma. 2013. 